\title{
Agencia social, sexualidad y embarazo en menores de 15 años*
}

\section{Agency, Sexuality and Pregnancy Under Age I5}

\section{Agência social, sexualidade e gravidez em menores de 15 anos}

Fecha de recepción: 22-06-15 Fecha de aceptación: 21-07-15 Disponible en línea: 01-11-15 doi: 10.11144/Javeriana.rgyps14-29.asse

Cómo citar este artículo:

Pacheco Sánchez CI. Agencia social, sexualidad y embarazo en menores de 15 años. Rev. Gerenc. Polít. Salud. 2015; 14(29): 62-82. http://dx.doi.org/10.11144/Javeriana.rgyps14-29.asse

El artículo utiliza datos generados en la investigación "Determinantes sociales de embarazo en menores de 15 años" que fue financiada en el marco de los convenios de cooperación 168/2011 y 036/2012 entre el Fondo de Población de las Naciones Unidas (UNFPA) y el Ministerio de Salud y Protección Social (MSPS) y donde el autor fue el investigador principal. Las conclusiones presentadas son exclusivamente del autor y no comprometen a las instituciones financiadoras. Es además, parte del trabajo de tesis doctoral en Sociología que actualmente está escribiendo el autor.

** Médico, especialista en Epidemiología, candidato a doctor en Sociología, investigador asociado de la Corporación para la Investigación Acción en Sociedad, Salud y Cultura (CISSC). Correo electrónico: ipacheco@cissc.org.co. Correspondencia: Carrera 25, Número 27A-37, Bogotá-Colombia. 


\section{Resumen}

El embarazo adolescente tiene un alto impacto socioeconómico. El objetivo de esta investigación fue interpretar los significados de la sexualidad y el embarazo de niñas menores de 15 años con experiencia de embarazo. Se realizó un estudio cualitativo interpretativo desde el enfoque fenomenológico de Alfred Schütz sobre el sentido común. Se realizaron 18 relatos de vida con adolescentes menores de 15 años con experiencia de embarazo en las ciudades de Bogotá, Cali, Cartagena y Medellín. Los significados de la menarquia, el inicio de relaciones sexuales, la unión en pareja, el uso de anticonceptivos y la atención del parto están influenciados por la agencia moral social e institucional. La empresa moral circula por los espacios de socialización de las adolescentes: madres, padres, hogares, instituciones y profesionales del sector de la salud y educativo clasifican, de acuerdo con su sentido común, lo que está bien o es "normal" para las niñas adolescentes.

Palabras clave: adolescentes; embarazo; sexualidad; moral; Colombia

\section{Abstract}

Teenage pregnancy has a high socioeconomic impact. The objective of this research was to interpret the meanings of sexuality and pregnancy of girls under 15 . An interpretive qualitative study from the phenomenological approach of Alfred Schütz over common sense was carried out. 18 life stories were written with girls under 15 years who had pregnancy experiences in the cities of Bogota, Cali, Cartagena and Medellin. The meanings of menarche, the sexual onset, marriage, contraceptive use and delivery care are influenced by the social and institutional moral agency. The moral enterprise circulates through the spaces of socialization of teenagers: mothers, fathers, homes, institutions and professionals in the health and education sector which classify according to their common sense what is right or "normal" for teenage girls.

Keywords: teens; pregnancy; sexuality; morality; Colombia

\section{Resumo}

A gravidez na adolescência tem impacto socioeconômico elevado. O objetivo desta pesquisa foi o de interpretar os significados da sexualidade e gravidez para meninas menores de 15 anos com experiência em gravidez. Estudo qualitativo interpretativo desde o enfoque fenomenológico de Alfred Schütz sobre o senso comum foi realizado. Fizeram-se 18 relatos de vida com adolescentes menores de 15 anos com experiência de gravidez nas cidades de Bogotá, Cali, Cartagena e Medellín. Os significados da menarca, o início de relações sexuais, a união em casal, o uso de contraceptivos e a assistência do parto são influenciados pela agência moral social e institucional. A empresa moral circula pelos espaços de socialização das adolescentes: mães, pães, famílias, instituições e profissionais do setor da saúde e educativo classificam, segundo o seu senso comum, aquilo que vai dar certo ou é "normal" para as meninas adolescentes.

Palavras-chave: adolescentes; gravidez; sexualidade; moralidade; Colômbia 


\section{Introducción}

El concepto de adolescente se popularizó a partir de los procesos de escolarización, dando lugar a un discurso alrededor de esta etapa de la vida que tiene como referente histórico la publicación del estudio de Stanley Hall en 1904, que describe la adolescencia como un periodo de tensiones e inestabilidad emocional en el que hay una ruptura con la infancia (1). Esta noción general aún hoy opera en las investigaciones e intervenciones dirigidas a esta población (2).

En años recientes, las investigaciones en neurociencias han reafirmado que la adolescencia es un periodo donde se da un rápido desarrollo cerebral que conduce a nuevas capacidades y comportamientos que activan las maneras de transitar en los contextos familiares, de pares, en ámbitos educativos y de la salud individual y colectiva (3). Es el tiempo donde se consolida el aprendizaje para el tránsito de la heteronomía a la autonomía. En el marco de los derechos humanos, las niñas, niños y adolescentes son sujetos con identidad, libertad y capacidad para tomar decisiones, iguales al resto de las personas. Viven un tiempo vital de desarrollo cerebral, desde la etapa prenatal hasta después de la pubertad, que combinado con la influencia y estimulación cultural proveen las condiciones para el desarrollo del libre albedrío (3-5).

En las definiciones sobre adolescencia subyace el interés de enfocar acciones hacia este grupo poblacional que se consolidó como sujeto de intervención durante la segunda mitad del siglo xx. Los temas de sexualidad han suscitado la atención, tanto de las ciencias biomédicas como de las ciencias sociales, desde hace más de cuatro décadas (2), y las investigaciones más específicas sobre fecundidad y embarazo tuvieron su auge en los Estados Unidos en la década de los setenta y en América Latina y el Caribe desde los ochenta (6).

En Colombia, en los últimos años el embarazo adolescente ha cobrado importancia por su magnitud e impacto social y económico. Según la última encuesta de demografía y salud, el 19,5\% de las adolescentes entre 15 y 19 años estaban embarazadas o tenían un hijo al momento de la entrevista (7). Aunque se muestra una estabilización del fenómeno, que venía creciendo en los últimos 15 años, las cifras siguen siendo altas para el país. Por otra parte, según la Organización Panamericana de la Salud (8), el 25\% de las mujeres en América Latina y el Caribe son madres antes de los 20 años de edad y el $45 \%$ de estos embarazos resultan del mal uso o de la falta de anticonceptivos. En colombianas entre 10 y 14 años, la razón de fecundidad se ha incrementado en los últimos años así: 2,74 por mil en el año 2000, 2,56 en el 2002, 2,73 en el 2004, 2,82 en el 2006 y 2,99 en el 2008, siendo con Brasil uno de los dos países con mayor razón de fecundidad en estas edades (9).

Las alarmas alrededor del embarazo adolescente se fundamentan en los impactos que genera, como: i) mayores riesgos de morbilidad y mortalidad para la madre y el recién nacido debido al escaso desarrollo físico y psicológico de la mujer (10); ii) aumento de la vulnerabilidad de los hijos a la violencia sexual, el maltrato infantil y la explotación sexual (11); iii) pobre potencial de progreso económico y social, a causa del truncamiento de la trayectoria formativa y la deserción escolar, lo que conlleva persistencia de bajo nivel educativo; y iv) limitaciones en la participación laboral futura y el desempleo. Todo ello contribuye a lo que se denomina "perpetuación del ciclo de la pobreza" y “feminización de la miseria”(10-12).

A pesar de existir acuerdos sobre causas e impactos del embarazo temprano, también 
coincidimos con Claudio Stern en que "hoy conocemos muy poco sobre el embarazo en la adolescencia [...] de que se trata de un fenómeno complejo y diverso — como prácticamente todos los fenómenos sociales-y de que habría que delimitar con mucho mayor rigor y precisión donde están los problemas ligados con el mismo, para quién o quiénes se constituyen en problemas, cuáles son sus determinantes [...]" (13). Siguiendo este planteamiento, estudiar el fenómeno de embarazo adolescente en menores de 15 años es clave para avanzar de manera adecuada en políticas, programas y proyectos efectivos para su prevención y la mitigación de los impactos bio-psico-sociales en las niñas que lo presentan.

Los determinantes estructurales que influyen en la salud de los y las adolescentes, como la clase social, la desigualdad económica y social y la educación (3), están también en la base de la ocurrencia del embarazo adolescente $(14,15)$. Este fenómeno se asocia a factores como la pobreza, el bajo nivel educativo y la deserción escolar que dificultan posteriormente la inserción en el mercado laboral y por ende la obtención de suficientes recursos económicos, y así se perpetúan estas trampas de pobreza (16-18). Varios autores consideran que la relación entre embarazo adolescente y pobreza debe leerse en forma inversa, no es el embarazo adolescente el que por sí mismo perpetúa la pobreza, sino las condiciones sociales, económicas y culturales las principales causas de una maternidad temprana (2,6). En este sentido, se critica el énfasis en la problematización del embarazo adolescente, cuando no se cuestionan las estructuras de desigualdad y vulneración en las que están inscritas las jóvenes y adolescentes (19). Stern (20) señala, de manera enfática, que las posibilidades de formación educativa y éxito laboral de esta población solo mejorarán con la modificación de tales estructuras. Otras investigaciones en con- textos de desplazamiento forzado muestran cómo la violencia estructural y las promesas incumplidas a las adolescentes en términos de sus derechos humanos son un marco proclive a la presentación del embarazo (21).

El embarazo adolescente está influenciado por la edad de inicio de las relaciones sexuales y la menarquía temprana (22-24). El inicio más temprano de las relaciones sexuales, sin embargo, no va acompañado de una unión más temprana ni del uso de anticonceptivos (25). El tipo y edad de inicio de la unión también se comportan como determinantes próximos del embarazo $(26,27)$. Se ha observado que el patrón de unión es clave para que el embarazo se lleve a término y se asuma la maternidad-paternidad $(15,28)$. La probabilidad de embarazo entre las unidas, formal e informalmente, es mayor, dada la tendencia a la no utilización de métodos anticonceptivos en tal situación (29-31). Esto se potencia por el poco conocimiento sobre salud sexual y reproductiva que tienen adolescentes y jóvenes (32-34), a lo que se suman las actitudes negativas hacia el uso de métodos anticonceptivos por parte de este grupo, como producto de la desinformación y mitos socioculturales para su uso o uso correcto $(10,22)$. Varios estudios han mostrado el menor uso y baja constancia en el uso de anticonceptivos entre adolescentes $(35,36)$, siendo menor en los sectores populares bajos, asociado a la vergüenza o al menor acceso por problemas económicos, y mayor entre las clases medias altas (37). También la baja percepción del riesgo hace que las/ los adolescentes no usen los métodos anticonceptivos, aunque los conozcan (38-40).

La insuficiente información, el desconocimiento y la escasa educación en materia de sexualidad y derechos sexuales y reproductivos constituyen otro factor determinante en la ocurrencia del embarazo adolescente (41-45), en conjunto con la presión y des- 
información sobre sexualidad ejercidas por los medios masivos de comunicación y los pares $(36,46)$.

Otra de las causas encontradas son las barreras de acceso a servicios de salud sexual y reproductiva y anticoncepción moderna, mucho más marcadas cuando se trata de menores de edad (47). Existen limitados servicios institucionales especializados en promoción de métodos de planificación familiar y salud sexual y reproductiva para adolescentes $(48,49)$. La edad a la que la legislación considera que un adolescente varón o mujer puede solicitar asesoría y/o provisión de métodos sin el acompañamiento de sus padres, se configura en una barrera de acceso a los servicios y por ende aumenta la probabilidad de embarazo en adolescentes que ya han iniciado relaciones sexuales (15).

Los estereotipos de género y las morales hegemónicas para hombres y mujeres hacen que el sexo seguro y el uso de métodos anticonceptivos se enfrenten a las ideas de feminidad y masculinidad: las mujeres buscan el amor romántico del "hombre ideal" y los hombres son impulsados a tener el máximo de parejas sexuales que puedan conseguir; los hombres piensan que son las mujeres las que deben utilizar los métodos anticonceptivos y ellas piensan que son ellos los que deben "saber" y orientar en estos elementos (50,51). Las vulnerabilidades en salud sexual y reproductiva y la probabilidad de concurrencia de eventos negativos se relacionan directamente con la divergencia existente entre la moral sexual para las mujeres y para los hombres (52,53). Estos elementos pesan mucho más cuando se trata de niñas y niños menores de 14 años, donde las representaciones hegemónicas dictan los comportamientos y prácticas en los contextos en que ellas y ellos viven y se desarrollan.
Varias investigaciones muestran cómo la presión de las parejas masculinas en el marco de relaciones asimétricas de género son determinantes en la presentación del embarazo. Las adolescentes acceden a tener relaciones sexuales, pero por modelos tradicionales de género muestran poca capacidad para negociar el uso de anticonceptivos $(36,40,54)$.

En este marco del conocimiento actual, este trabajo tiene como objetivo interpretar desde las subjetividades de las niñas menores de 15 años con experiencia de embarazo, construidas a partir del sentido común hegemónicas, las significaciones de su experiencia con la sexualidad y el embarazo.

\section{Materiales y métodos}

Este artículo se centra en un estudio de tipo cualitativo interpretativo a través de 18 relatos de vida (55), de adolescentes menores de 15 años embarazadas o con experiencia de embarazo, de las ciudades de Bogotá, Cali, Cartagena y Medellín, guiados por la mayor presentación de embarazos en estas cuatro ciudades y en los estratos socioeconómicos bajos, según las bases de datos del DANE en el periodo 2005-2009. Los relatos se obtuvieron entre finales del año 2011 y comienzos del 2013. La procedencia de las adolescentes fue de sectores rurales y urbanos populares. La localización de los casos se realizó a través de direcciones territoriales de salud y/o instituciones públicas prestadoras de servicios de salud.

El protocolo de investigación se sometió a valoración y fue aprobado por un comité de ética, constituido ad hoc por el Fondo de Población de Naciones Unidas (UNFPA) y el Ministerio de Salud y Protección Social (MSPS). Adicionalmente, tuvo que ser sometido a los comités de ética de las instituciones hospitalarias que contribuyeron 
en la identificación de los casos. ${ }^{1}$ El estudio está clasificado como de riesgo mínimo, según los estándares internacionales y la Resolución 008430 de 1993 del Ministerio de Salud de Colombia, dado que la entrevista de relato de vida aborda aspectos sensibles del comportamiento humano y se requirió el consentimiento escrito informado de las adolescentes, acogiendo el precepto de la Corte Constitucional de Colombia referido a que "La jurisprudencia constitucional ha reconocido en los menores la titularidad del derecho al libre desarrollo de la personalidad y la posibilidad de consentir tratamientos e intervenciones sobre su cuerpo, aun cuando tengan un carácter altamente invasivo. En esta medida, descarta que criterios de carácter meramente objetivo, como la edad, sean los únicos determinantes para establecer el alcance del consentimiento libremente formulado por los menores para autorizar tratamientos e intervenciones sobre su cuerpo. En materia de aborto el legislador, si lo estima conveniente, podrá establecer reglas específicas en el futuro sobre representación, tutela o curatela sin menoscabar el consentimiento de la menor de catorce años" (56). En aquellos casos en que la adolescente accedió a la realización del relato de vida, pero supeditó su participación a la autorización de la madre/padre o tutor responsable, se pidió también consentimiento informado a esta/este.

Partiendo del supuesto de que muchas de estas adolescentes estuvieron expuestas a violencia sexual, la entrevista para el relato de vida se asumió como un acto reparador

1 Esto hizo que el trabajo de campo se extendiera en el tiempo, ya que las aprobaciones tuvieron distintas fechas, lo que prolongó el periodo de recolección de los datos. Según la legislación nacional, si el protocolo fue aprobado en un comité de ética, se entiende aprobado para sus efectos en el territorio nacional; sin embargo, las instituciones hospitalarias entienden que cualquier acción en la que participen debe pasar por su propio comité de ética. del sujeto de derechos, por lo cual no se centró en el trauma o el recordar el hecho de violencia. Previamente a la realización de los relatos de vida, se estableció contacto con los servicios de salud a los que estaban afiliadas las adolescentes seleccionadas, para asegurar que si aparecía un caso de abuso sexual en la realización del relato de vida, la institución de salud acogiera a la adolescente y le prestara los servicios correspondientes, de acuerdo con el modelo de atención integral en salud a víctimas de violencia sexual del msps.

En este trabajo, el relato de vida se entiende en la tradición etnosociológica desarrollada por el sociólogo francés Daniel Bertaux: "Hay un relato de vida desde el momento en que un sujeto cuenta a otra persona, investigador o no, un episodio cualquiera de su experiencia vivida. El verbo 'contar' (narrar) es aquí esencial: significa que la producción discursiva del sujeto ha adoptado una forma «narrativa»" (55). El episodio sobre el que las adolescentes narran su experiencia es el embarazo, y sobre este se construye el relato con elementos antecedentes y consecuentes. La construcción de la explicación del fenómeno no se da por generalización estadística, sino por generalización sociológica en la cual "la generalización de los hallazgos puede presumirse toda vez que el análisis descansa en aquellos recursos del sentido común que indexan la normalidad que prevalece entre los sujetos estudiados" (57), y además porque relacionando los varios testimonios sobre una experiencia vivida —en este caso el embarazo y el parto- de una situación social, se superan sus singularidades para lograr, mediante una construcción progresiva, una representación sociológica de los componentes colectivos de la situación narrada-estudiada (55).

Las categorías que se tuvieron en cuenta en este trabajo y sobre las que se organizó la narración y el análisis posterior tienen que 
ver con la experiencia corporal y social de la menarquia, la iniciación sexual, la unión, el uso de anticoncepción moderna y la atención del parto.

Las entrevistas fueron transcritas, procesadas como base de datos textuales y analizadas según las categorías deductivas definidas y otras inductivas que aparecen en el lenguaje del sentido común narrado. Para el análisis se realizó un proceso hermenéutico, con el fin de explorar el sentido común y los significados que este tiene para las adolescentes en función de la experiencia vivida desde la menarquia, pasando por inicio de su ejercicio sexual, la unión o no, el embarazo y el parto, e identificar las principales determinaciones de orden social y cultural que influyen en la forma en que construyen esos significados.

La perspectiva analítica usada es la fenomenológica, que parte de la conceptualización que hace Alfred Schütz del sentido común como el conjunto de saberes prácticos acumulados a lo largo de las generaciones y que heredamos (ya están ahí cuando nacemos y simplemente somos socializados dentro de ese conocimiento), que nos permiten saber qué es el mundo y ubicarnos en él de forma inmediata. Se trata de un conjunto de recetas y tipificaciones que utilizamos rutinariamente en la vida cotidiana, lo que nos proporciona una sensación de seguridad y de transparencia de la realidad (58). Epistemológica y metodológicamente, se prioriza la voz de las protagonistas en la investigación, esto es, de las adolescentes menores de 15 años con experiencia de embarazo.

\section{Resultados}

Los relatos de vida se concentraron en sectores marginales y populares, donde no son ajenas las nociones de desigualdad e iniquidad. ${ }^{2}$ En este apartado se priorizan los factores que parten de la subjetividad de las adolescentes que han compartido momentos de sus vidas y se hará referencia, según el caso, a elementos estructurales de la economía, la cultura y la biología.

\section{El sentido común de la menarquia}

La presentación de la menarquia se relaciona directamente con los niveles de desarrollo, principalmente con las mejoras en nutrición, y su tendencia ha sido a una ocurrencia cada vez más temprana. En Europa se reconoce una media para la menarquia de 11,5 años, y en América Latina hay autores que sostienen que ocurre entre los 12 y los 14 años (9). Este fenómeno, asociado al inicio de relaciones sexuales, aumenta el riesgo de presentación temprana de embarazos en menores de 15 años.

¿A qué edad te desarrollaste?

A los 11 años.

¿Pero tú sabías todo lo del desarrollo?

Sí, aunque cuando yo me desarrollé me asusté. Me dijeron que me cuidara, pero no me dijeron que podía salir embarazada. [Adolescente 1, Cartagena]

\section{¿A los cuántos años te desarrollaste?}

A los 10. Pues yo estaba en piscina y tenía mi vestido de baño y yo siento que algo se me sale. Vi sangre y le dije a mi tía que qué era eso y me dijo que me había desarrollado. Ahí en la piscina ella me compró una toalla y nos fuimos para la casa y le dije a mi mamá y me compraron más toallas y me explicaron cómo era.

¿Y te explicaron que podías quedar en embarazo después de eso?

No. Hubo un tiempo en que mi mamá sî me dijo que podía quedar embarazada,

2 Se toma aquí el sentido literal dado por la Real Academia Español (RAE) de "maldad, injusticia grande". 
pero solamente eso. Que si tenía relaciones podía quedar embarazada. [Adolescente 3, Cartagena]

La mayoría de las niñas entrevistadas tuvieron su menarquia antes de los 12 años, lo que muestra que forman parte de la tendencia mundial al adelantamiento de este hecho biológico. Por tratarse de un evento que está ligado al desarrollo social y económico, la tendencia de adelanto de la menarquia no puede ser modificada. Sin embargo, lo que ocurre con las niñas que experimentan la menarquia más temprana sí puede tener desenlaces diferenciales en distintos contextos sociales. Como se observa en los testimonios, las niñas fueron informadas sobre la menarquia en relación con los elementos de higiene y la incorporación del sentido común del "cuidado", sin crear capacidades para la toma de decisiones sobre su cuerpo y su sexualidad. La menarquia se significa con la limpieza, la higiene corporal y en ocasiones en relación con el embarazo - a la reproducción-, pero no se construyen sentidos sobre la sexualidad, su ejercicio y sus consecuencias positivas y/o negativas. Están ausentes las referencias a la asunción del cuerpo como territorio propio y autónomo, no se discuten los derechos reproductivos ni los derechos sexuales y tampoco existen elementos para que las niñas aprendan sobre el abuso y la violencia sexual. Estos tópicos no fueron abordados en la familia ni en la escuela.

\section{El inicio de las relaciones sexuales}

La edad de iniciación sexual de las adolescentes, según los relatos de vida, está próxima a la menarquia. Esto podría explicarse por los contextos de precariedad y pobreza, donde el inicio de las relaciones sexuales es más temprano. El escaso diálogo que existe en el medio social en que crecen las adolescentes, sobre el campo de la sexualidad, hace que las niñas no conozcan información básica y, lo que es más complejo, no tengan elementos mínimos para una toma de decisiones informada y sin presiones, sobre todo en relaciones asimétricas en edad y conocimientos, como ocurre con la mayoría de relatos de vida realizados.

¿A qué edad comenzaste a tener relaciones?

Sí, a los 13 y a los 13 y medio fue que quedé embarazada.

¿Cuántos compañeros sexuales has tenido en tu vida?

No, pues mi novio anterior y este.

¿Y de qué edad era tu novio anterior?

De los mismos años que él, de 17 años.

[Adolescente 4, Bogotá]

¿A qué edad tuviste tu primera relación sexual? $\mathrm{A} \operatorname{los} 12$.

¿Y con cuántas parejas has tenido relaciones? Una. Sí, porque yo lo conocí a él cuando tenía 12 y él tenía 18 . Y a los dos años quedé embarazada.

¿En el colegio había clases sobre eso?

No, nunca. [Adolescente 5, Bogotá]

¿Cuándo fue tu primera relación sexual?

A los 13 años.

¿Y fue con el papá de tu hija?

Sí.

Yen ese momento, isabías que podías quedar en embarazo?

No, no sabía que podía quedar en embarazo.

[Adolescente 2, Cartagena]

Una investigación que utilizó relatos de vida en adolescentes bogotanas menores de 14 años, mostró cómo el sentido común del amor romántico está aún presente en la cultura colombiana y las madres y adultas referentes de las niñas promueven una noción del "cuidado de los hombres" como un grupo genérico, pero en el cual hay siempre una excepción que es "el hombre de la vida" (53). Por lo tanto, podemos pensar que las adoles- 
centes sujetas del embarazo mantienen este ideal del amor romántico y responden a las exigencias de su primera o segunda pareja para iniciar relaciones sexuales muy temprano en el noviazgo o con la unión temprana.

\section{La unión en el marco social de la precariedad}

En América Latina, y en Colombia en particular, según los datos disponibles, la edad mediana de la unión en pareja no ha tenido cambios, pasando de 20,8 años en 1986 a 20,9 años en el 2010 (15). Sin embargo, existe una marcada desigualdad cuando observamos el mismo dato por nivel educativo: la unión en el grupo de mujeres de 25 a 29 años con educación superior se da después de los 26 años, mientras en las jóvenes sin educación formal la mediana de la edad de la primera unión es 17 años (15). En los relatos de vida de este trabajo, la unión de las menores de 15 años estuvo muy por debajo de la mediana nacional. La unión puede ser desencadenante de las relaciones sexuales y el embarazo temprano, o la presentación de este último imponer la unión.

Después de que pasaron los meses comenzamos a tener relaciones, entonces él me dijo que me fuera con él. Entonces me fui con él, pero yo no quería, después dije que sí y me fui con él. Cuando estábamos juntos a mí me fueron a buscar, entonces me vine otra vez.

¿Al cuánto tiempo de tener relaciones con él quedaste embarazada?

Como a los tres meses.

¿Pero tú ya vivías con él?

Sí. Llevaba un mes con él.

¿Por qué decidiste irte a vivir con él?

No sé. Él me lo propuso y yo me fui con él.

Me dijo "vamos, yo te llevo para mi casa". Yo le dije que no, y después me volvió 70 a decir y le dije que sí. [Adolescente 1, Cartagena]
Estuvimos juntos y quedé embarazada, pero no me di cuenta y él tampoco sabía. Entonces yo me fui a vivir a una finca y él se fue a vivir a otra parte, entonces pasó el tiempo y yo me di cuenta que estaba embarazada, me hice la prueba y salió positiva. Toda mi familia me ha apoyado. [Adolescente 1, Cali]

\section{¿Y te colabora con algo?}

No, y no quiero que me colabore. No quiero nada que venga de él. Lo que me llevé fue una decepción. Yo le dije a él antes de venirme que yo asumía todo yo sola, que no necesitaba que me ayudara en nada. Y mi papá también dijo lo mismo. [Adolescente 4, Cartagena]

En los relatos aparecen las múltiples posibilidades: la niña engañada por la pareja que tenía otra familia y una edad mucho mayor de la que le contó y que ahora ha decidido ser madre sola (Adolescente 4, Cartagena), o la de los novios que se separan y luego del embarazo y el parto vuelven a ser pareja, pero sin convivir, y ella continúa viviendo con sus padres (Adolescente 1, Cali). También se observa una tendencia a la unión que precede al embarazo, sugerida o presionada por los varones, para que la chica vaya a vivir a casa de los suegros, pero, conocida la noticia del embarazo, las niñas retornan a su casa materna, y con ellas, sus parejas masculinas. Finalmente, está el embarazo como desencadenante de la unión, y que concluye con la pareja viviendo en la casa de alguno de sus progenitores, los de ella o los de él.

En los relatos de las adolescentes entrevistadas, aun cuando muchas de ellas están escolarizadas, se evidencian las pocas posibilidades de negociación de la unión y de las relaciones sexuales. Elementos estructurales como las desigualdades de género y edad, donde los varones proponen y presionan la unión y/o las relaciones sexuales, en contextos de vulnerabilidad y precariedad, inciden 
para que las niñas acepten sin que tengan la información, las capacidades y habilidades para negarse. En otros casos, las presiones familiares y la violencia en el interior conducen a la unión como forma de escape.

\section{Decisiones anticonceptivas y la agencia social hegemónica}

El uso de métodos anticonceptivos en las adolescentes transita por múltiples facetas. Una es la adolescente menor que comienza a planificar antes de las relaciones sexuales, e incluso antes de emparejarse; esa decisión se da en un contexto de servicios de salud "amigables", pero los efectos secundarios y el cambio subsecuente de método crean una ventana donde aparece el embarazo, porque su pareja no utiliza protección.

Entonces yo me puse a planificar con la pasta, yo me conseguí un novio, el papá de él. Él también estudiaba. Entonces pues me convenció a tener relaciones. Yo seguía planificando normal, porque la jefe de allá es muy amable y todo. Yo planifiqué como 8 o 9 meses con la pasta. Como me caía muy mal, entonces la jefe me dijo que yo la podía cambiar. Entonces la jefe dijo que con las inyecciones, entonces yo me aplicaba las inyecciones. Y ahí fue que quedé en embarazo porque todo fue muy rápido. No me venía el período y a mí nunca se me atrasaba, entonces fuimos con mi mamá a hablar con la jefa y ella me mandó un examen y ahí fue que salí embarazada. Entonces fue entre las pastas y la inyección, no las podía cambiar. Porque yo tuve que esperar para la inyección como tres o cuatro meses. Cuando me aplicaron la inyección ahí fue que quedé embarazada. Yo me di cuenta a los tres meses que me tocaba otra vez la inyección. [Adolescente 3, Cali]

Es en el periodo de transición y cambio de los métodos anticonceptivos (MAC), que ante la falta de oportunidad en la provisión del nuevo método, se produce el embarazo. A pesar de la existencia de un servicio y una profesional amigable, las dificultades de contar y proveer el nuevo MAC hacen que una adolescente autónoma termine con un embarazo no planeado. El servicio amigable promueve el uso de MAC entre las niñas, pero no el uso del condón por sus parejas, reforzando el imaginario cultural de que la anticoncepción es cosa de mujeres y perdiendo la oportunidad de promover la doble protección.

De otro lado, el uso de anticonceptivos en mujeres unidas precozmente es impulsado en algunos casos por suegras y/o madres, pero los mitos sobre las consecuencias de la anticoncepción moderna y las barreras de acceso a servicios de salud hacen que las adolescentes prefieran tener relaciones sexuales sin protección anticonceptiva; tampoco tienen el empoderamiento para exigir el uso del condón a su pareja y, por lo tanto, se exponen al embarazo.

\section{¿Tú me decías que cuando uno usaba anticonceptivos podía quedar estéril? \\ $\mathrm{Si}$, pastillas, inyecciones. \\ $Y$ cuando tú tuviste el bebé, ¿te hablaron de esas cosas? \\ No. Porque eso fue antes de que yo quedara embarazada. Porque mi suegra me dijo que me inyectara para no salir embarazada. Pero yo no quería porque podía quedar estéril. [Adolescente 1, Cartagena]}

El imaginario dominante de la responsabilidad femenina del control de la fecundidad hace que los varones y sus referentes familiares (en este caso, la suegra) no asuman el uso del condón como una posibilidad de prevención del embarazo y de infecciones de trasmisión sexual. La falta de conocimiento a profundidad también es otra de las facetas que se evidencian en los relatos de las adolescentes que han iniciado relaciones 
sexuales, como en el caso de esta adolescente cartagenera, de 13 años, con su pareja de 17 años: "¿Nunca escuchaste hablar de métodos anticonceptivos? Hasta que estaba embarazada fue que me enteré que habían muchas cosas para uno cuidarse" [Adolescente 2, Cartagena].

El conocimiento, como unos de los elementos necesarios para la toma de decisiones, se combina también con la voluntad de "formar una familia", como en este caso, en el cual una adolescente de 13 años y su pareja utilizan un método anticonceptivo y ella lo suspende para quedar embarazada, en aparente consenso con su pareja, aunque la primera referencia es sobre el deseo de él, seguido por la "recomendación"-“orden" del varón de la pareja para suspender el método:

\section{Cuando supiste que estabas embarazada y le contaste al papá, ¿qué dijo? \\ Se puso feliz. Él quería tener un bebé. \\ ¿O sea que lo estaban buscando los dos? \\ Sí. Habíamos hablado que queríamos formar una familia. \\ ¿Y quieren tener más? \\ Sí, por ahí en unos cuatro años. \\ ¿Y cómo haces ahora para no quedar embarazada?}

Cuidarme. Voy al médico y me manda una inyección.

¿Y alguna vez la habías usado?

Sí. Cuando empecé a salir con él.

¿Y luego la suspendiste?

Sí, él me dijo que no la usara más porque quería tener familia. La suspendí y ahí quedé embarazada. [Adolescente 3, Cartagena]

Interrogada hoy, cuando el bebé tiene cuatro meses, sobre si estuvo bien embarazarse tan joven, ella reconoce que debió esperar un poco "porque es importante tener un trabajo para poder atender a los hijos. Yo tengo que pedirles a mis papás o a mis suegros". La condición material a la que están sometidas estas jóvenes antes y después del embarazo hace que resignifiquen su decisión, aunque todo lo que le pasa con sus hijos "les gusta". El contexto rural de donde proviene esta niña, nos habla del patrón tradicional de unión temprana, embarazo temprano y el deseo de aparente consenso de formación de familia, pero donde el varón siempre tiene la última palabra.

De otra parte, está el caso de la niña que a sus 11 años tiene su primera relación sexual, en un contexto marcado por las desigualdades en edad, género, conocimientos y en los procesos de maduración biológica y psicológica, ya que su pareja tenía 18 años y estudiaba en una universidad bogotana. Aquí también se relata un supuesto consenso y el uso de condón que falla, sin explicación clara.

\section{¿Usaste algún método anticonceptivo?}

Usé condón pero no sé por qué sucedió esto.

Era la primera vez y quedé embarazada.

[Adolescente 4, Cartagena]

Niñas que inician relaciones sexuales tempranamente, como esta adolescente cartagenera, o como la adolescente de Valle del Cauca que tiene su primer hijo a los 11 años y el segundo a los 14, tienen experiencias marcadas por relaciones de afecto en contextos de desigualdad y violencia. Hombres mayores en edad, maduración biológica y psicológica y con más conocimiento y experiencia en sexualidad, que utilizan las necesidades de afecto de las niñas como elemento de presión para tener relaciones sexuales y producen embarazos, abandonando posteriormente a las niñas. Las adolescentes han adquirido escasos elementos para la agencia social necesaria sobre sí mismas y sobre otros para ejercer sus derechos de autonomía y autodeterminación sobre su cuerpo y su sexualidad, y sus familias y la sociedad en su conjunto no las están protegiendo. 
A pesar de la claridad que se puede observar sobre el abuso en casos como los relatados, los derechos de las niñas no se han garantizado. En ninguno de los dos casos reportados de las niñas de 11 años se prestó atención en salud mental y las dos fueron atendidas como cualquier madre en los servicios de salud, sin ubicarlas en la ruta de atención integral de la violencia sexual. De hecho, en el caso de la adolescente del Valle del Cauca, con su segundo parto en Medellín a los 14 años, la garantía de no repetición y todas las acciones necesarias para protegerla y restituirle sus derechos no se realizaron, lo que demuestra la incapacidad y fracaso de la institucionalidad para actuar en la garantía de los derechos de las niñas.

Finalmente, encontramos casos en que las niñas, habiendo iniciado relaciones sexuales consensuadas y periódicas, no quieren quedar embarazadas, comunican a sus padres el deseo de usar anticonceptivos y esa opción les es negada:

Con él, siempre fue con él. Pues se dieron las cosas cuando mi mamá nos comenzó a dejar solos, a dar mucha confianza. Mi mamá le cogió mucha confianza a él. Se comenzaron a dar las cosas, quedé embarazada. Y ahí sí le dolió. Porque mi papá no me dejó planificar, que porque yo era muy pequeña y yo no hacía eso, que yo estaba muy pequeña para hacer eso [...] Yo le dije a mi mamá que yo quería planificar. ¿Cuántos años tenías? Pues 13 años. Yo les dije a mi papá y a mi mamá que quería planificar. Mi mamá me dijo que sí, pero mi papá me dijo que no, que yo estaba muy pequeña, que yo no podía tener relaciones sexuales. Que porque eso era malo para mí, que no me iba a poner a planificar. Pues yo seguí con lo mismo, y él no me hizo caso. Cuando quede embarazada ahí si me regañó. Se puso bravo conmigo. Pero yo no tenía la culpa, yo se lo dije a ellos dos y pues mi mamá no contó con el apoyo de mi papá.

[Adolescente 1, Bogotá]

Pues yo al principio, o sea, no, no lo quería tener, pero pues de todas maneras ella no tenía la culpa porque es la responsabilidad de uno y uno tiene que asumirla, ¿no? Entonces pues y de todas maneras él no me iba a dejar tampoco, entonces decidimos tenerlo, entonces asumir la responsabilidad, ino? [...] ¿Y tú por qué no lo querías tener?

No, porque pues primero era muy joven y no, no, no sé $[\ldots]$

¿Tú cuando empezaste a tener relaciones sexuales hablaste con tu pareja de usar anticonceptivos, algún método anticonceptivo? No, pues nosotros nos cuidábamos con el preservativo y yo una vez le dije a mi mami que si me dejaba poner la inyección y me dijo que no. [Adolescente 5, Bogotá]

Estos dos casos, el primero de una niña de 13 años con pareja de 14 años y la segunda, una niña 14 años con pareja de 20 años, con inicio de relaciones sexuales a los 13 y 12 años, respectivamente, previeron consecuencias y solicitaron a sus padres ayuda para iniciar la anticoncepción, y aunque los mismos padres aprobaban las relaciones de noviazgo y propiciaban contextos para iniciar y mantener relaciones sexuales, negaron la posibilidad de anticoncepción. Las adolescentes por sí solas no recurrieron a ningún servicio de salud, posiblemente porque en la mayoría de ellos aún se mantiene la idea de negar asesoría en salud sexual y reproductiva (SSR) a niñas que no vienen acompañadas de sus padres.

\section{Parto: de la agencia moral profesional a la violencia obstétrica}

En los lugares de atención del parto se dan casos de vulneración de derechos en las niñas. En estudios realizados en México se ha reportado como: "A los hospitales públicos las mujeres acceden en su calidad de ciuda- 
danas, porque es su derecho. La atención que ahí reciben, sin embargo, es vivida en términos despersonalizantes y la posibilidad de reclamar sus propios derechos sexuales y reproductivos (i.e. trato de ciudadana y no de paciente) se ve reducida al mínimo o eliminada" (59). En el caso de las niñas, esto es aún más grave, pues no son vistas como ciudadanas, sujetos de derechos, sino como menores incapaces de tomar decisiones y de interpretar adecuadamente las instrucciones de los profesionales y técnicos en salud. Por tanto, el sentido común profesional hace que se obligue, se amenace y se culpabilice de cualquier resultado negativo en el proceso del parto-puerperio. La agencia moral se mezcla con el "saber técnico" de los y las profesionales que dirigen o ayudan a las adolescentes durante el proceso del parto, dándole una imagen de neutralidad y cientificidad.

\section{¿Cómo te trataron los médicos y las enfermeras? Cuando estaba en cirugía las enfermeras me criticaron. Que estaba muy pequeña y hablaban muchas cosas, pero a mí me daba igual todo. [Adolescente 4, Cartagena]}

Porque un doctor me vio y me dijo que si yo seguía así, yo no podía tener el bebé natural, que tocaba hacerme una cesárea, porque yo estaba muy chiquita para estar teniendo un bebé. Y el doctor me regaño prácticamente [...] Me dijeron puje fuerte, pero tenía que respirar, me decían, no se vaya a quedar pujando porque se le puede ahogar el bebé, entonces yo pensé solo en pujar. El doctor me decía puje fuerte, si puja fuerte yo le doy agua. Y yo con esa sed que tenía, pues dije a pujar. [Adolescente 4, Bogotá]

La crítica, el regaño y la culpabilización por los malos resultados que pudieran ocurrir, se combina con la presión respecto a necesidades básicas de las niñas como la deshidratación causada por el trabajo de parto.
De allí, en ocasiones, se pasa a situaciones extremas de vulneración de sus derechos como sujetarlas y amenazarlas.

Pues yo sentí muchas ganas de pujar, pujaba y pujaba. Cuando el doctor me fue a hacer el tacto, pues la niña tenía la cabeza por fuera, $\mathrm{y}$ los doctores se asustaron. Me tuvieron que entrar muy rápido, tenía muchas ganas de pujar, pero los doctores me decían que no pujara. Y pues me entraron, me iban a amarrar los pies porque les daba miedo que yo cerrara las piernas y yo les dije a los doctores que no, que no me amarraran los pies que yo sabía que yo podía resistir. El doctor me dijo, le vamos a desamarrar las piernas pero si la niña se muere es culpa suya, yo le dije al doctor que bueno. [Adolescente 1, Bogotá]

Y cuando ya iba dilatada en 9, me acuerdo que un doctor $[. .$.$] , que era todo brusco$ llamó a una enfermera para que me hiciera el tacto y ya iba en 9. Y él me despertaba y me decía, boba o estúpida. Y cuando ya iba en 9, yo estaba abierta de piernas porque me dijeron que no las podía cerrar y llego y me hizo el tacto pero duro. Y yo le dije: pero ya me lo hicieron. Y él dijo: ay quítese que usted no es la doctora. Y como todas las enfermeras se iban, la que me acompaño ahí fue una aseadora, que ella era la que me decía que tranquila que eso era duro pero que tenía que seguir. Ósea la que me daba alientos. [...] Y yo le dije: es que a mí me da miedo porque usted tiene la mano muy brusca, dijo: de malas, a mí que me importa, quien la mando a meter las patas. [Adolescente 6 , Bogotá]

El saber técnico se combina con habitus adquiridos de las profesiones (57) y con la moralidad de las matrices culturales de donde provenimos. La sexualidad "no correcta", sin importar si es en el marco del abuso o de la decisión propia, se vuelve punible por el círculo inmediato de la atención. Los profe- 
sionales y técnicos se convierten en jueces y ejecutores de la pena por la falta cometida. Las adolescentes que se embarazan y llegan a los servicios de salud y son sometidas a estos malos tratos, los resienten, pero no tienen la capacidad para denunciar o exigir sus derechos, ya que aceptan lo que ocurre en la institución hospitalaria como parte del castigo que la sociedad instituye para la falta cometida.

\section{Discusión y conclusiones}

Existen elementos estructurales que se reflejan en los relatos de vida y son coincidentes con las cifras de embarazo adolescente en menores de 15 años en el país. Existe una relación importante entre pobreza, desigualdad e inequidad que se expresa en estas niñas que se embarazan antes de los 15 años. Como plantean Stern (14) y la Cepal (15), el embarazo en niñas adolescentes está ligado a la desigualdad económica y social, trabajar sobre este aspecto es prioritario. Sin embargo, a veces se confunde o se invierte el corolario que es necesario seguir, estableciéndose que posponer el embarazo adolescente, por sí solo, disminuirá la pobreza, desconociendo que este es un fenómeno multicausal y que si no actuamos sobre el resto de causas difícilmente se avanzará en la reducción de la pobreza. En un Estado social de derecho, es deseable garantizar los derechos de las y los adolescentes, para que en esa medida pospongan la unión, el primer hijo/a y que permanezcan en la escuela, sin asumir responsabilidades que les nieguen posibilidades de desarrollo. La prevención del embarazo en niñas adolescentes debe contener acciones que combatan la desigualdad de manera integral. Como lo muestran los estudios basados en encuestas y censos poblacionales, cuanto menor desigualdad, menor presentación de embarazo adolescente.
Otro de los elementos estructurales que se evidencian en este estudio tiene que ver con las violencias que atraviesan los contextos y las vidas de estas niñas. Los relatos dan cuenta de la violencia estructural social y política que está presente en forma de desplazamiento, violencia sexual y violencia de género. La violencia estructural de orden sociocultural, representada en las desigualdades de poder basadas en edad y género, es la constante. Al revisar la base de datos de embarazos del quinquenio 2005-2009, se observó cómo solo en un $1 \%$ las parejas de las madres adolescentes menores de 15 años del país eran pares entre 11 y 14 años. En un 48,5\% se trataba de hombres de 15 a 20 años, y un $36,2 \%$, entre 21 y 30 años. El $6,32 \%$ eran hombres mayores de 40 años y se presentaron 10 casos de varones mayores de 70 años (60). La violencia se expresa en vulneraciones que van desde el abuso sexual, la coerción, la presión económica, las asimetrías, el desconocimiento y la experiencia, etc. El embarazo de menores de 15 años con padres mayores nos habla de unas masculinidades patriarcales hegemónicas, de las cuales este fenómeno es apenas una expresión más.

La menarquia se ha adelantado en la mayoría de países, incluido Colombia. Sin embargo, en un sentido común social sobre sexualidad de las adolescentes, relacionada con la agencia moral que ejercen las personas referentes adultas, la llegada de la primera menstruación no es utilizada para informar y formar en sexualidad. La mayoría de niñas entrevistadas fueron informadas sobre la menarquia en relación con los elementos de higiene y el sentido común del "cuidado", sin crear capacidades y habilidades para protegerse. A la menarquia se le da significado con relación a la reproducción, de una manera genérica en la advertencia de "a partir de ahora cuidado con los hombres", pero no se construyen significados y sentidos sobre la sexualidad, los derechos reproductivos y los 
derechos sexuales, el placer, el cuidado del cuerpo y la autonomía en las decisiones. El desarrollo de capacidades para el cuidado del cuerpo, el ejercicio de derechos, la prevención del abuso y la violencia sexual están ausente en los ámbitos familiares y escolares.

La edad de iniciación sexual de las adolescentes, en los relatos de vida, está próxima a la menarquia. Esto podría explicarse por los contextos de precariedad y pobreza, donde el inicio de las relaciones sexuales es más temprano y se agudiza con el fenómeno de adelantamiento del desarrollo biológico expresado en la edad de la menarquia. Sin embargo, esto no puede generalizarse al universo de las adolescentes. En Colombia, como en otros países de América Latina, la edad promedio del inicio de la actividad sexual, en las mujeres de la cohorte de 25 a 29 años incluidas en las encuestas de demografía y salud, pasó de 19,4 años en 1986 a 18,2 años en el 2010, con diferenciales importantes por nivel educativo, ya que en las que refirieron que no tenían educación, la edad mediana de inicio fue de 14,9 años, las de primaria 16 años, las de secundaria 17,1 y las de superior 18 años como edad mediana de la iniciación sexual (15). Si se toma como proxy de pobreza y desigualdad el bajo nivel educativo, este podría explicar el comportamiento del grupo estudiado, donde el inicio en contextos de pobreza y desigualdad es más temprano.

La tendencia que se reconoce para Colombia en el adelanto de las relaciones sexuales es más evidente si se revisa el dato de las mujeres entre 20 y 24 años que se habían iniciado sexualmente a las edades exactas de 15, 18 y 20 años. El porcentaje de mujeres de 15 años que había iniciado relaciones sexuales a esta edad pasó de $6,1 \%$ en 1986 a $13,8 \%$ en 2010; en las de 18 años cambió de 30,9 a 76 60,3\% y en las de 20 años pasó de 49 a 82,2\% (61). El porcentaje de las mujeres de 15 años que inició las relaciones sexuales se duplicó entre 1986 y 2010 , aumentando en un 7,7\%.

Esta tendencia está en correspondencia con la que muestran estudios sobre la modernidad sexual en países desarrollados: adelanto de las relaciones sexuales, que cada vez más antecederán a las uniones, combinado con la convergencia de las edades de iniciación entre hombres y mujeres (62). Sin embargo, estamos aún lejos de las afirmaciones del sentido común —del público en general, de los medios de comunicación y de algún sector de la Academia- que hablan de relaciones sexuales tempranas generalizadas en la adolescencia. Cecilia Gayet y Patricio Solis, en México, han planteado cómo esas ideas, que provienen del manejo incorrecto de indicadores o de una lectura apresurada y no razonada de los resultados científicos, producen un nuevo sentido común que hace que los debates y las políticas no se enfoquen adecuadamente. En el caso particular del inicio de las relaciones sexuales, ellos afirman: "Diferentes artículos utilizan la edad media de inicio sexual de los sexualmente activos en un determinado grupo de edades (por ejemplo, los sexualmente activos de 15 a 19 años) obtenida a partir de distintas encuestas, medida inadecuada porque por una parte deja fuera del análisis a aquellos que no han experimentado relaciones sexuales y por otra se ve afectada por los cambios en la estructura de edades al interior del grupo en cuestión, situación que afecta particularmente a sociedades en rápido cambio demográfico, como es el caso de México. Para subsanar estos problemas pueden utilizarse medidas más adecuadas, como la proporción de los sexualmente activos a una edad exacta, o la edad de inicio sexual calculada a partir de tablas de vida" (63). Esto ocurre de manera similar en Colombia.

Podemos adscribir estas características descritas por Bozon sobre la sexualidad contempo- 
ránea a grupos urbanos y educados de estratos altos en Colombia y en otros países de América Latina, en donde se observa una tendencia en la cual existe una convergencia en el inicio de la actividad sexual entre las jóvenes de mayor y menor educación y "simultáneamente una clara divergencia en la edad al casarse y al tener hijos, que se va profundizando a medida que aumenta el nivel educativo" (15).

En cambio, las iniciaciones sexuales en el grupo de mujeres que compartieron sus historias en este trabajo corresponden más bien a patrones socioculturales tradicionales marcados por el abuso derivado de las relaciones de poder desiguales, basadas en género, edad, sexo y clase social. Este patrón sociocultural tradicional coexiste con el modelo de modernidad sexual truncada propuesto por Rodríguez y Openhayn (64), en sectores urbanos pobres y vulnerables (podríamos decir populares) donde el acceso a medios de comunicación, la presión social de pares y referentes, la moda y la necesidad de estar al día en sus tendencias, contrasta con una escasa posibilidad de autonomía en la toma de decisiones sobre el cuerpo y la sexualidad y con marcos donde el amor romántico, las carencias afectivas, la presión de pares, la violencia y el abuso sexual, se combinan con la no aceptación de las adolescentes como sujetos sexuales en sus contextos familiares y sociales. La presión de las personas referentes, principalmente madres, padres y maestros/as, tiene que ver con la generación de un sentido común sobre la sexualidad y la maternidad. El ensalzamiento de la maternidad como el proyecto de "las mujeres" y el de la "hombría" ligada al inicio y multiplicidad de relaciones sexuales en los hombres comienza mucho antes de la adolescencia y genera contradicciones con los discursos hegemónicos morales sobre la negación de la sexualidad adolescente.
En los relatos de vida de las adolescentes también se evidenciaron las pocas posibilidades de negociación de la unión en un contexto de elementos estructurales como las desigualdades de género y edad, donde los varones proponen y presionan la unión y/o las relaciones sexuales, y las niñas, en contextos de vulnerabilidad, aceptan, sin que tengan la información, las capacidades y las habilidades para negarse. En otros casos, las presiones familiares y la violencia en el interior de los hogares conducen a la unión como forma de escape.

El uso de métodos anticonceptivos modernos antes del embarazo, en este estudio, fue casi nulo, ya sea porque las adultas y adultos referentes - madres, padres, suegras- no permitían el uso, o por la existencia de barreras en el acceso a las consultas de anticoncepción y/o asesoría en salud sexual y reproductiva, o en la oportunidad de provisión de los métodos, que como muestran algunos autores, se cruza con la exigencia de que las personas jóvenes sean acompañadas por un adulto, por la desorganización del sistema o por problemas en los sistemas de referencia y contra-referencia (47).

En este sentido, es necesario empezar a dilucidar la confusión que se presenta en lo que se refiere a "la capacidad de toma de decisiones en el ámbito legal y de salud" (65) o, por lo menos, iniciar su discusión en los ámbitos de los servicios de salud. Aunque, desde la perspectiva legal en Colombia, el parámetro para otorgar la capacidad de toma de decisiones en lo relativo a relaciones sexuales es la edad (mayores de 14 años), en salud la capacidad de toma de decisiones tiene una mayor complejidad y está acorde a la variabilidad de las poblaciones; esto quiere decir que la capacidad para tomar decisiones depende de varios factores que no están determinados únicamente por la edad. Como es conocido, en el caso Gillick 
en Gran Bretaña, ocurrido a mediados de la década de los ochenta, la Corte británica estableció que un profesional podía proveer anticoncepción a menores de 16 años que lo solicitaran, sin el consentimiento previo, ni el conocimiento de sus padres o madres (66). La Corte valoró la complejidad para que una persona adolescente pudiera consentir (voluntad) su propio tratamiento médico, en función de tener la inteligencia (razonamiento para prever consecuencias) y el conocimiento para entender el problema al que se enfrenta, comprendiendo la naturaleza y los efectos de los procedimientos que se sugieren por el personal de salud $(65,66)$.

Estos elementos fueron resumidos en los criterios de Fraser, derivados de la sentencia Gillick, así: i) el/la joven va a entender el consejo del profesional; ii) el/la joven no se puede convencer para informar a sus padres; iii) el/la joven es probable que comience o continúe teniendo relaciones sexuales, con o sin tratamiento anticonceptivo, a menos que reciba el tratamiento indicado; iv) a menos que reciba tratamiento anticonceptivo, la salud física o mental de la persona adolescente, o ambos, son propensos a sufrir, y los mejores intereses del o la joven le obligan a recibir consejos sobre anticoncepción o tratamiento con o sin consentimiento de los padres (66). Es necesario que la norma sobre abuso sexual para toda menor de 14 años no quite la posibilidad del ejercicio de la autonomía a estas adolescentes, sobre todo para efectuar labores de prevención y promoción de los derechos sexuales y los derechos reproductivos. Estudiar y aplicar los criterios de Fraser en las asesorías de salud sexual y reproductiva de adolescentes, aun menores de 15 años, puede redundar en la prevención del embarazo e ITs y en la promoción de los derechos sexuales y derechos reproductivos.

78 En otros desarrollos que contribuyen a esta discusión, el Relator Especial sobre el Dere- cho a la Salud de la onU, en su informe anual de 2009, afirmó como: "la Convención sobre los Derechos del Niño exige que se respete la evolución de las facultades del niño y que se dé la debida importancia a sus opiniones en función de su edad y su madurez. ${ }^{3}$ Se deben dar a conocer ampliamente los riesgos y beneficios de las intervenciones médicas al niño y, si éste posee suficiente madurez, solicitar su consentimiento informado (67). ${ }^{4}$

Adicionalmente a estos planteamientos del orden jurídico internacional, es necesario revisar el hecho de que además de la edad y la etapa del desarrollo de las personas adolescentes, existe una influencia de las propias experiencias biográficas en el desarrollo de la autonomía y la capacidad de tomar decisiones $(65,68)$; un ejemplo es que los adolescentes que han sido víctimas de deterioro social, enfermedades crónicas, trabajo infantil, cuidado de hermanos/as, etc., pueden ser más maduros en una determinada situación que implique decisiones, comparados con sus pares de una misma edad cronológica. En el mismo sentido, Climent (69) plantea cómo en los estilos parentales educativos de características democráticas, los y las adolescentes están más expuestos/as a aprendizajes sobre toma de decisiones que en los estilos autoritarios, permisivos o negligentes, y esto podría brindarles capacidades mayores para la toma de decisiones, comparativamente con los/las demás.

En este marco internacional y nacional, se plantea como tesis para discusión, que vista la concentración de embarazos en los trece y catorce años, la garantía de derechos de estas niñas debe circular por una valoración profunda de las vulnerabilidades y riesgos

3 Artículo 5 y párrafo 1 ) del artículo 12 de la Convención sobre los Derechos del Niño.

4 Artículos 3, 13 y 17 de la Convención sobre los Derechos del Niño; Convención sobre los Derechos del Niño, Observación general núm. 3, párr. 20. 
para abuso sexual, pero también por una valoración desde el sector salud de su nivel de desarrollo y su capacidad para la toma de decisiones que permita el ejercicio de sus derechos a la salud sexual y reproductiva. Es un hecho que la situación recurrente de la adolescente menor de 14 años que consulta para anticoncepción, porque teme quedar embarazada, y la misma menor en la fila del control prenatal dos o tres meses después, no se puede seguir presentando por la ambigüedad moral de los prestadores de servicios y por el imperativo legal que se aduce para no asesorar y proveer en anticoncepción y SSR.

Por otra parte, a pesar de la existencia y defensa de la norma sobre que toda relación con menores de 15 años es abuso, en dos de los casos registrados en niñas de 11 años, y a pesar de la claridad que se puede observar sobre el abuso en estos casos, la garantía de derechos de las niñas no ha sido realizada. Ninguna de las dos recibió atención en salud mental y las dos fueron atendidas como cualquier madre en los servicios de salud. De hecho, en el caso de la adolescente del Valle del Cauca, con su segundo parto en Medellín a los 14 años, la garantía de no repetición y todas las acciones que se debieron realizar para protegerla no se efectuaron, lo que demuestra la incapacidad y el fracaso de la institucionalidad para actuar en la garantía de los derechos de las niñas.

Lo que se evidencia como tesis central de este trabajo es que la agencia moral social e institucional es una constante. La empresa moral (70) circula por los espacios de socialización de los adolescentes: madres, hogares y otras instituciones que regulan la sexualidad adolescente.

Profesionales del sector educativo y de la salud clasifican, de acuerdo con su sentido común, lo que está bien o es "normal” para las niñas adolescentes. La crítica constante a adolescentes embarazadas, la prescripción de conductas ("lo que pasó, pasó"; "tú no puedes hacer eso"; "la bebecita no tiene la culpa de tu error"), los llamados a la equivocación, al error, a la culpa de las adolescentes por no haber seguido el proyecto de vida definido por la sociedad, aunque el contexto social y económico lo hagan imposible, son los elementos que marcan el sentido común hegemónico. Se le sigue dictando la conducta como "niña" incapaz de decidir, pero se la juzga y culpa como adulta para que asuma su responsabilidad por el error cometido.

En el sector salud, la atención de las adolescentes en el control prenatal y el parto se cruza con vulneraciones de diverso grado de sus derechos humanos. La crítica, el regaño y la culpabilización por los malos resultados que pudieran ocurrir se combinan con la presión respecto a necesidades básicas de las niñas, como la deshidratación causada por el trabajo de parto. De allí, en ocasiones, se pasa a situaciones extremas de vulneración de los derechos de las niñas, como sujetarlas y amenazarlas. El saber técnico se combina con el habitus adquirido de las profesiones a través del currículo oculto (59) y con la moralidad de las matrices culturales de donde provenimos. La sexualidad "no correcta", sin importar si es en el marco del abuso o de la decisión propia, se vuelve punible por el círculo inmediato de la atención. Los profesionales y técnicos se convierten en jueces y ejecutores de la pena por la falta cometida. Las adolescentes que se embarazan llegan a los servicios de salud y son sometidas a estos malos tratos, los resienten, pero no tienen la capacidad para denunciar o exigir sus derechos, ya que aceptan lo que ocurre en la institución hospitalaria como parte del castigo que la sociedad instituye para la falta cometida.

Estas adolescentes están sujetadas por una sociedad que no las reconoce y que crea normas para protegerlas, que el sentido común 
vuelve normas para limitarlas. La tensión entre autonomía y ejercicio de la sexualidad está inscrita en los intersticios de una sociedad conservadora que no las protege, que no las resarce cuando son vulneradas y que las culpa del ejercicio de su sexualidad, cuando eligen ejercerla y cuando son víctimas de abuso.

\section{Referencias bibliográficas}

1. Dávila-León O. Adolescencia y juventud: de las nociones a los abordajes. Última Década. 2004; 12 (21): 89-104.

2. Adaszko A. Perspectivas socioantropológicas de la adolescencia, la juventud y el embarazo. En: Gogna M, editor. Embarazo y maternidad en la adolescencia. Estereotipos, evidencias y propuestas para políticas públicas. Buenos Aires: Cedes-Unicef; 2005. pp. 33-59.

3. Viner-Russell M, Ozer EM, Denny S, Marmot M, Resnick M, Fatusi A, Currie C. Adolescence and the social determinants of health. Lancet. 2012; 379: 1641-52.

4. Evers K. Neuroética. Buenos Aires: Katz; 2010.

5. Sigrid V, López-Peña P, Saric D. Salud sexual y reproductiva para jóvenes. Revisión de evidencia para la prevención. Washington: BID; 2013.

6. Pantelides EA. Aspectos sociales del embarazo y la fecundidad adolescente en América Latina. Notas de Población. 2004 diciembre; 78 (31): 7-33.

7. Profamilia. Encuesta nacional de demografía y salud (ENDS) 2010. Bogotá: Profamilia; 2010.

8. Organización Panamericana de la Salud. Salud sexual y reproductiva del jóven y el adolescente. Oportunidades, enfoques y opciones. Washington: OPs; 2008.

9. Gómez PI, Molina R, Zamberlin N. Factores relacionados con el embarazo y la maternidad en menores de 15 años en América Latina y el Caribe. Lima: Promsex; 2011.

10. Flórez CE. Factores socioeconómicos y contextuales que determinan la actividad reproductiva de las adolescentes en Colombia. Rev Panam Salud Pública. 2005; 18 (6): 388-402.

11. Parada-Baños AJ, Becerra-Cornejo DA, VillacisVallejos CE. Embarazo en la adolescencia. En: Universidad Nacional de Colombia. Obstetricia integral. Bogotá: Siglo XXI; 2009.

12. Baeza-W B, Póo-F AM, Vásquez-P O, Muñoz-N S, Vallejos-V C. Identificación de factores de riesgo y factores protectores del embarazo en adolescentes en la novena región. Rev Chil Obstet Ginecol. 2007; 72: 76-81.

13. Stern C. Significado e implicaciones del embarazo adolescente en distintos contextos socioculturales de México: reseña de un proyecto en proceso. Estudios Sociológicos. 2003 sep-dic; 21 (63): 725-45.
14. Stern C. El "problema" del embarazo en la adolescencia. Contribuciones a un debate. México DF: El Colegio de México; 2012.

15. Comisión Económica para América Latina y el Caribe (Cepal). Panorama actual y perspectivas futuras de la fecundidad en América Latina. En: Panorama social de América Latina. Santiago de Chile: Cepal; 2011. pp. 82-105.

16. Henao-Escovar J, González C, Vargas-Trujillo E. Fecundidad adolescente, género y desarrollo. Evidencias de la investigación. Territorios - Universidad de los Andes: Cider. 2007; 16 (17): 47-70.

17. Salazar A, Rodríguez LF, Daza, RA. Embarazo y maternidad adolescente en Bogotá y municipios aledaños. Consecuencias en el estudio, estado civil, estructura familiar, ocupación y proyecto de vida. Persona y Bioética (Universidad de la Sabana). 2007; 11(29): 170-85.

18. Silva-Árias A, González-Román P, Torres-Rodríguez M. Consideraciones teóricas y empíricas acerca de la fecundidad adolescente. Rev Fac Cienc Econ. 2008; 16 (2): 47-59.

19. Irvine J. Culture difference and adolescent sexualities. In: Irvine J, editor. Sexual cultures and construction of adolescent identities. Filadelfia: Temple University; 1994. pp. 3-28.

20. Stern C. El embarazo en la adolescencia como problema público: una visión crítica. Salud Pública Mex. 1997; 39 (2): 137-47.

21. Nieto J, Rincón L, Ávila S, Mariño Y, Forero M. Aproximación a los significados de la paternidad, la maternidad y el embarazo adolescente, en contextos de desplazamiento en la Localidad de Ciudad Bolívar en Bogotá. Bogotá: SDS-UNFPA; 2011.

22. Lion K, Prata N, Stewart C. La maternidad adolescente en Nicaragua: una evaluación cuantitativa de factores asociados. Perspectivas Internacionales en Salud Sexual y Reproductiva. 2010; (Número especial): 16-21.

23. Flórez CE, Soto VE. Fecundidad adolescente y desigualdad en Colombia y la Región de América Latina y el Caribe. Santiago de Chile: CeladeDivisión de Población y UnFPA; 2006.

24. León P, Minassian M, Borgoño R, Bustamante F. Embarazo adolescente. Rev Ped Elec [internet]. 2008; 5 (1): 44.

25. Rodríguez-Vignoli J. Reproducción adolescente y desigualdades en América Latina y el Caribe: un llamado a la reflexión y a la acción. Madrid: oIj; 2008.

26. Rodríguez J. High Adolescent Fertility in the Context of Declining Fertility in Latin America. Nueva York: United Nation Expert Group of Meeting on Adolescents, Youth, and Development (Eclac); 2011.

27. Palomino-Gamarra L, Ascate-K`ana GM. Documento técnico. Análisis de situación de salud de las y los adolescentes. Ubicándolos y ubicándonos. Lima: Ministerio de Salud; 2009.

28. Binstock G, Pantelides EA. La fecundidad adolescente hoy: diagnóstico sociodemográfico. En: Gogna M, coordinador. Embarazo y maternidad en la adolescencia. Estereotipos, evidencias y propuestas para políticas públicas. Buenos Aires: 
Cedes-Ministerio de Salud y Ambiente de la Naciòn-Unicef; 2005. pp. 77-112.

29. Aquino EML, Heilborn ML, Knauth D, Bozon M, Almeida MC, Araújo J et al. Adolescência e reprodução no Brasil: a heterogeneidade dos perfis sociais. Cad Saúde Pública. 2003; 19 (2): 377-88.

30. Remez L, Singh S, Prada E, Rosero-Bixby L, Bankole A. Asegurar un mañana más saludable en Centroamérica: proteger la salud sexual y reproductiva de la juventud de hoy. Nueva York: Guttmacher Institute; 2008.

31. Remez L, Singh S, Prada E. Trends in Adolescent Union and Childbearing in Four Central American Countries. Población y Salud en Mesoamérica. 2009 jul-dic; 7 (1): 1-20.

32. Barker P, Guthrie K, Hutchinson C, Kane R, Wellings K, editores. Teenage Pregnancy and Reproductive Health: Summary Review. Londres: Royal College of Obstetricians and Gynaecologists; 2007.

33. Pallitto C, Murillo V. Childhood Abuse as a Risk Factor for Adolescent pregnancy. El Salvador. J Adolesc Health. 2008; 42 (6): 580-6.

34. Haldre K, Rahu K, Rahu M, Karro H. Individual and familial factors associated with teenage pregnancy: an interview study. Eur J Public Health. 2009 jun; 19 (3): 266-70.

35. Peláez-Mendoza J. Salud sexual y reproductiva de adolescentes y jóvenes: una mirada desde la óptica de los derechos humanos. Rev Cuba Obstet Ginecol [internet]. 2008 may-ago; [acceso: agosto del 2012]; 34 (2). Disponible en: <http://bvs.sld.cu/revistas/ gin/vol34_2_08/gin05208.htm > .

36. Despacho de la Primera Mujer de Medellín, compilador. Memoria del trabajo realizado por la Red de Prevención del Embarazo Adolescente. Años 2004, 2005 y primer semestre de 2006. Medellín: Red de Prevención del Embarazo Adolescente; 2006.

37. Núñez-Urquiza RM, Hernández-Prado B, GarcíaBarrios C, González D, Walker D. Embarazo no deseado en adolescentes y utilización de métodos anticonceptivos posparto. Salud Pública Méx. 2003 jun; 45 (1): 92-102.

38. González JC. Conocimientos, actitudes y prácticas sobre la sexualidad en una población adolescente escolar. Rev Salud Pública Bogotá. 2009 feb; 11(1): 14-26.

39. Torriente-Barzaga N, Diago-Caballero D, RizoVázquez AC, Menéndez-López LR. Conocimientos elementales sobre educación sexual en alumnos de una escuela secundaria básica urbana. Rev Haban Cienc Méd. 2010 nov; 9 (4): 576-87.

40. Näslund-Hadley E, Manzano G. Educación de calidad: ¿un anticonceptivo eficiente? Aportes de BID (Banco Interamericano de Desarrollo). 2011dic; 12: 1-4.

41. Alfonso M. Girls Just Want to Have Fun? Sexuality, Pregnancy, and Motherhood among Bolivian Teenagers. Nueva York: Inter-American Development Bank; 2008. Research Department Working paper; 615.
42. Gubert D, Faganello-Madureira VS. Iniciação sexual de homens adolescentes. Ciência e Saúde Coletiva. 2009 jul-ago; 14 (4): 1119-28.

43. Ávila-Montes GA, Barbena-García M, ArguelloMunguía P. Factores de riesgo asociados al embarazo en adolescentes en comunidades marginales de Comayagüela. Rev Méd Hondur. 2011 abr.-jun; 79 (2): 68-72.

44. Contreras-Rodríguez S, Martín-Cortes D. El embarazo en la Adolescencia. ¿Un problema social en Santa Clara, Villa Clara, Cuba? Contribuciones a las Ciencias Sociales [serie en internet] abril 2011. [Acceso: agosto del 2012]. Disponible en: www. eumed.net/rev/cccss/12/

45. Barinas I. Voces de jóvenes. Una aproximación a la realidad juvenil, a partir del estudio del significado y las implicaciones del embarazo en adolescentes, según género y contextos socioculturales en la República Dominicana. República Dominicana: Mixtli Grafika; 2012.

46. Zelaya EY, Coto-García JR. Factores socioculturales que condicionan el embarazo adolescente en los municipios de Intibucá y Jesús de Otoro, departamento de Intibucá. Revista Población y Desarrollo-Argonautas y Caminantes. 2011; 7 (7): 47-55.

47. Montoya P, Barreto E, Blanco AJ, Cárdenas Y, Chavarro C, Piñeros S. Barreras y facilitadores para el acceso de adolescentes a los servicios de salud sexual y reproductiva en Bogotá. En: Mejía ML, Pacheco CI, Muñoz LM, editores. Embarazo adolescente en Bogotá: construir nuevos sentidos y posibilidades para el ejercicio de derechos. Bogotá: SDS-UNFPA; 2011. pp. 153-220.

48. Martín-Alfonso L, Reyes Díaz Z. Conducta sexual, embarazo y aborto en la adolescencia. Un enfoque integral de promoción de salud. Rev Cubana Salud Pública [internet]. 2003 abr-jun; 29 (2).

49. Alonso V. Las organizaciones de la sociedad civil: entre la prevención, la asistencia y la promoción de derechos. En: Gogna M, coordinador. Embarazo y maternidad en la adolescencia. Estereotipos, evidencias y propuestas para políticas públicas. Buenos Aires: Cedes-Unicef; 2005. pp. 215-49.

50. Gogna M, Ramos S. Lay beliefs and gender stereotypes: Uncknowledged risks for sexually transmitted diseases. Paper presented at the Conference Reconceiving Sexuality: Internacional Perspectives on Gender, Sexuality and Sexual Health. Río de Janeiro, Apr. 14-17, 1996.

51. Stern C. Estereotipos de género, relaciones sexuales y embarazo adolescente en las vidas de jóvenes de diferentes contextos socioculturales en México. Estud Sociol /México. 2007; 35 (1): 105-29.

52. Szasz I. Sexualidad y género: algunas experiencias de investigación en México. Debate Fem. 1998 oct; 18 (9): 77-104.

53. Pacheco-Sánchez CI, Rincón-Suárez LJ, Guevara EE, Latorre-Santos, C, Enríquez-Guerrero C, Nieto-Olivar JM. Significaciones de la sexualidad y salud reproductiva en adolescentes de Bogotá. Salud Pública Mex. 2007; 49: 45-51. 
54. Cortés-Ortiz ME, Valencia-Micolta SG, VásquezTruissi ML. "Mi mente decía no... mi cuerpo decía sí...": embarazo en adolescentes escolarizadas. Avances en Enfermería. 2009 jul-dic; 27 (2): 57-70.

55. Bertaux D. Los relatos de vida. Perspectiva etnosociológica. Barcelona: Ediciones Bellaterra; 2005.

56. Colombia. Corte Constitucional. Sentencia C-355 de 2006 [internet]. Disponible en: http://www.corteconstitucional.gov.co/relatoria/2006/C-355-06.htm.

57. Castro R. Teoría social y salud. Buenos Aires: Editorial-CRIM; 2011.

58. Schütz A. El problema de la realidad social. Buenos Aires: Amorrortu; 1974.

59. Castro R. Habitus profesional y ciudadanía: hacia un estudio sociológico sobre los conflictos entre el campo médico y los derechos en salud reproductiva en México. En: Castro R, López-Gómez A, editores. Poder médico y ciudadanía: el conflicto social de los profesionales de la salud con los derechos reproductivos en América Latina. Avances y desafíos en la investigación regional. Montevideo: Universidad de la República; México DF: UnAM, CRIM; 2010. pp. 49-72.

60. Pacheco-Sánchez CI, Mejía-Gómez ML, MartínezParra AG, Moreno-López DJ. Determinantes sociales de embarazo en menores de 15 años. Bogotá: UNFPA; 2014 [internet]. Disponible en: http://unfpa. org.co/wp-content/uploads/2015/02/sm-Determembarazo-menores-15-años.pdf.

61. Rodríguez-Vignoli J. Situación del embarazo a edad temprana y en adolescentes en América Latina y el Caribe, y sus implicaciones para el desarrollo evolutivo de los niños, niñas y adolescentes. En: Seminario Internacional Buenas Prácticas y Lecciones Aprendidas para el Diseño de un Modelo de Intervención para la Prevención y Atención del Embarazo a Edad Temprana y en Adolescentes Adolescentes; 2012 abr 26-27; Caracas; CeladeDivisión de Población de la Cepal [acceso: agosto del 2012]. Disponible en: <http://www.idena.gob. ve/images/pdf/Ponencias/b-1_jrodriguez.pdf $>$.

62. Bozon, Michel. A quel âge les femmes et les hommes commencent-ils leur vie sexuelle? Comparaisons mondiales et évolutions récentes. Population et Sociétés. 2003 juin (391).

63. Gayet C, Solís P. Sexualidad saludable de los adolescentes. Salud pública Méx. 2007: 49 (supl 1); 47-51.

64. Rodríguez J, Openhayn M. Maternidad adolescente en América Latina y el Caribe. Tendencias, problemas y desafíos. Desafios. 2007; (4): 4-9.

65. Ministerio de Salud. Gobierno de Chile. Guía práctica de Consejería para adolescentes y jóvenes. Orientaciones generales. Santiago de Chile: 2011.

66. Bird S. Children and adolescents - who can give consent? [internet]. Aust Fam Physician. 2007, mar; 36 (3):165-6.

67. ONU Asamblea General. Informe del Relator Especial sobre el derecho de toda persona al disfrute del más alto nivel posible de salud física y mental, Anand Grover. 200910 ago; un Doc A/64/272; 15-6.

68. Alderson, Priscilla. Competent children? Minors' consent to health care treatment and research. Soc Sci Med. 2007, dic; 65 (11), 2272-83.

69. Climent GI. Representaciones sociales sobre el embarazo y el aborto en la adolescencia: perspectiva de las adolescentes embarazadas. Cuadernos FHyCS-Unju. 2009; (37): 22-42.

70. Freidson E. La profesión médica. Barcelona: Taurus; 1970. 